\title{
AN INFRARED STUDY OF SOUTHERN Be STARS: GROUND-BASED AND IRAS OBSERVATIONS
}

\author{
P. Persi and M. Ferrari-Toniolo \\ Istituto Astrofisica Spaziale, CNR, CP. 67 \\ 00044 Frascati, Italy
}

\section{Introduction}

Infrared observations represent a powerful tool in understanding the physical conditions of the circumstellar matter around $\mathrm{Be}$ stars. Previous IR studies have been reported by different authors (i.e. Gehrz et al. 1974, Persi et al. 1982, Ashok et a1. 1984, and Waters, 1986).

For this purpose, we have undertaken an IR study of 21 southern Be stars including JHKLM photometry, CVF spectra at resolution $\mathrm{R}=100$ around the IR HI emission lines $B r \gamma, B r \alpha$, and $P f \gamma$, and IRAS data at 12 and 25 microns taken from the Point Sources Catalogue. The IRAS flux densities were corrected following the procedure described in the IRAS Explanatory Supplement.

The near-IR observations were obtained in March 1985 at the $1 \mathrm{~m}$ ESO (La Silla, Chile) telescope, equipped with the InSb Spectrophotometer. In the next section we discuss the nature of the observed IR excesses, and from the measured Bra/Bry intensity line ratios for three Be stars, we derive the mass-1oss rates.

\section{Infrared Excesses}

In order to derive the infrared excesses from our sample of $\mathrm{Be}$ stars, the unreddened spectral points were compared with appropriate Kurucz (1979) model atmospheres. In Fig. 1, we show an example of IR energy distributions obtained for the Be stars HD 50013 and HD 110432 . The solid line in the figure represents the stellar continuum, while the dashed line shows the derived IR excess continuum. From this analysis we found that $\sim 60 \%$ of our observed stars have an IR excess greater than the stellar continuum already in the near-IR ( $\sim 2-4$ microns), while the remaining $B e$ stars show a slight IR excess only at longer wavelengths $(\geq 12 \mu \mathrm{m})$. For the first Be stars, the IR excess continuum is well fitted by a power law spectrum $S_{\nu} \sim \nu^{\alpha}$ with $\alpha$ IR $=0.6-0.9$. This result could be interpreted in terms of $f f+b f$ emission from a hydrogen ionized enyelope with an electron density of the type $n=n_{0}(r / r) 2-2.5$ with $\mathrm{n}=10^{11}-10^{32} \mathrm{~cm}^{-3}$. In addition, the luminosity of the envelope L(IR) obtained integrating the IR excesses, seems to be well correlated with the stellar luminosity with $L(I R) / L^{*}=5 \times 10^{-3}$. 
IR HI Emission Lines

All the Be stars of our sample were observed at the Bry line $(2.167 \mu \mathrm{m})$. Six of these were also observed at the $B r \alpha(4.054 \mu \mathrm{m})$ and $\mathrm{Pf} \gamma$

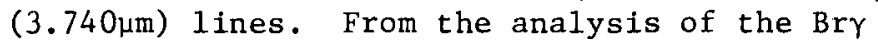
lines we found that only a small fraction $\left(\sim 10^{-5}\right)$ of the stellar energy that is incident on the envelope, is converted to Bry emission, in agreement with the result obtained by Persson and MacGregor (1985), obtained analyzing the Bro line from a different sample of Be stars.

The measured intrinsic $\mathrm{Br} / \mathrm{Br} \gamma$ line ratios of $0.64,0.43$ and 0.45 relative to the Be stars HD 50013, HD 91465 and HD 105435, indicate, according to the theory of Simon et al. (1983), that the lines are formed in an outflowing wind. Therefore, from the observed $B r \alpha$ line intensity, and applying the model of Simon et al. (1983), we estimate the mass loss rates for these three Be stars. The values are, respectively, $10^{-7}$, $7.5 \times 10^{-8}$ and $2 \times 10^{-8} \mathrm{M}_{\odot} / \mathrm{yr}$.

A detailed analysis of the IR HI emission lines including the hydrogen optical lines observed simultaneously (Giovannelli et al. 1986) is in progress.

In conclusion 13 Be stars of our sample show a strong IR excess already at $\lambda \geq 2 \mu$ and are well fitted up to $25 \mu$ with a power spectral law. This excess is interpreted as due to $\mathrm{fftbf}$

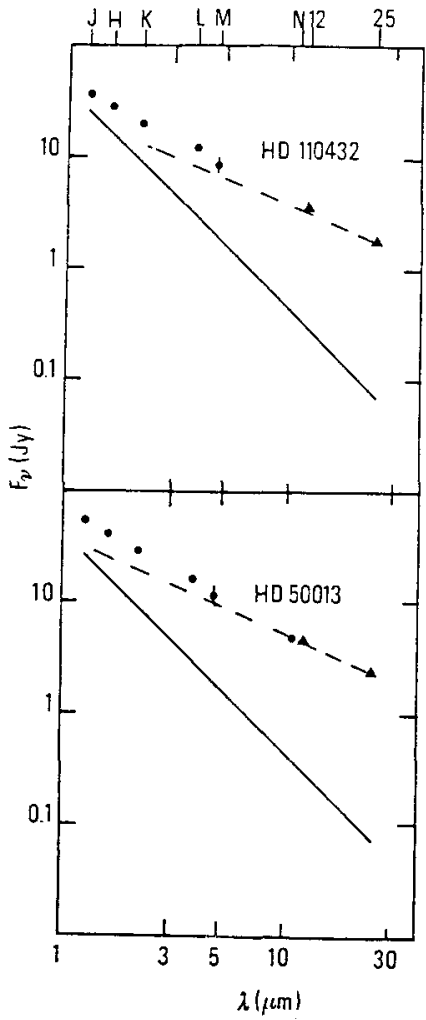

Fig. 1. IR energy distribution of HD 50013 and HD 110432. emission from a moderately expanding high density envelope. The analysis of the IR HI emission lines is very promising, and we suggest that observations at higher spectral resolution with infrared gratings now available be made.

\section{References}

Ashok, N. M. et al. (1984), Mon. Not. R. Astr. Soc., 211, 471. Gehrz et al. (1974), Astrophys. J., 191, 675.

Giovannelli, F. et al. (1986), these proceedings. Kurucz, R. L. (1979), Astrophys. J. Suppl., 40, 1. Persi et al. (1982), IAU Symp. No. 98, "Be Stars", p. 247, (Reidel Publ. Co.).

Persson, S. E., McGregor, P. J. (1985), Astron. J., 90, 169.

Simon, M. (1983), Astron. J., 266, 623.

Waters, L. B. F. M. (1986), Astron. Astrophys., 162, 121. 


\section{DISCUSSION FOLLOWING PERSI}

Henrichs:

Is there any information available about IR variability of the stars in your sample?

Persi:

Yes, several stars in our sample were importantly observed with the same instruments by Dr. Dachs two or three years before. Anyway, we have indications of IR variability of $\gamma$ Cas , X Per and o Cas observed by us from 1979 up to now with a monthly frequency. 